\title{
XII Russian Conference "Current Problems of Petrochemistry" (with international participation) dedicated to the memory of S. N. Khadzhiev
}

XII Russian Conference "Current Problems of Petrochemistry" dedicated to the memory of S. N. Khadzhiev will be held on October 5-9, 2021, at the Groznyi State Oil Technical University, Groznyi.

\section{Organizers}

Russian Academy of Sciences (RAS) Joint Scientific Council of the RAS on the Chemistry of Oil, Gas, Coal, and Biomass A. V. Topchiev Institute of Petrochemical Synthesis, Russian Academy of Sciences N. D. Zelinsky Institure of Organic Chemistry, Russian Academy of Sciences Groznyi State Oil Technical University

Organizing Committee

Chairman Corresponding Member (RAS) A. L. Maksimov

\section{Program Committee}

\section{Co-Chairmen}

Academician (RAS) S. M. Aldoshin Academician (RAS) V. P. Ananikov O. A. Baulin Academician (RAS) A. G. Dedov Corresponding Member (RAS) U. M. Dzhemilev Academician (RAS) Z. R. Ismagilov Professor V. M. Kapustin Professor E. A. Karakhanov Corresponding Member (RAS) V. A. Likholobov
Academician (RAS) V. I. Bukhtiyarov Academician (RAS) M. P. Egorov

Professor A. M. Mazgarov

M. Sh. Mintsaev Academician (RAS) V. N. Parmon

K. B. Rudyak Corresponding Member (RAS) V. F. Razumov Academician (RAS) O. G. Sinyashin Corresponding Member (RAS) S. D. Varfolomeev R. S. Yarullin

\section{Scientific Program}

The scientific program of the Conference will include plenary lectures (45 min), key reports (30 min), oral reports (15 $\mathrm{min}$ ), and posters on the following subjects:

- Petrochemistry and Oil Refining;

- Gas Chemistry, Carbon dioxide Utilization, and Hydrogen Production and Storage;

- Alternative and Renewable raw Materials;

- Processing of Coal and Polymer Wastes.

Contacts: A. V. Topchiev Institute of Petrochemical Synthesis of the RAS, 29 Leninsky prosp., 119991 Moscow, Russian Federation; e-mail: conference-2020@ips.ac.ru

Elena Mikhailovna Zakharyan, phone: +7 917543 1122; e-mail: Zakharyan@ips.ac.ru;

Natal'ya Nikolaevna Petrukhina, phone: +7 916204 2738; e-mail: n.petrukhina@ineos.ac.ru.

\section{http://www.conference.forenewchemistry.ras.ru}

